\title{
Virtual Manufacturing Cell Rescheduling Considering Multiple Strategy Combinations and Machine Failures
}

\author{
Wenmin $\mathrm{Han}^{1, *}$, Zhe Lv ${ }^{1}, \mathrm{Na} \mathrm{Lv}^{1}$ \\ ${ }^{1}$ Institute of Economics and Management, Jiangsu University of Science and Technology, Zhenjiang, Jiangsu, China \\ *Corresponding author. Email: wlmh63@163.com
}

\begin{abstract}
In terms of rescheduling problems under machine failure, this paper proposes a virtual manufacturing cell rescheduling method based on the combination of multiple strategies. According to the systematic analysis of the cost factors of rescheduling, the cost-evaluation function of the rescheduling scheme is constructed. Then the virtual manufacturing cell rescheduling model is constructed with the goal of minimizing the maximum completion time and the machine adjustment deviation, and which is solved by NSGA- II algorithm. The result of the numerical experiment shows that the combined applicable of multiple rescheduling strategies is beneficial to maintain the applicability of the rescheduling scheme under different disturbance situations, improve rescheduling efficiency, reduce disturbance deviation, and reduce disturbance repairing costs.
\end{abstract}

Keywords: Virtual manufacturing cell, Rescheduling method, Machine failure, NSGA-II.

\section{INTRODUCTION}

With the gradual development of economy and society, multi-variety and small-size batch production mode has become the mainstream production mode of current enterprises. Some enterprises have adopted cell manufacturing to achieve timely response to market demand. However, most of the manufacturing enterprises' machines are large, heavy, difficult or unable to move. Therefore, with the implement of this production mode, enterprises need to pay a higher replacement cost. As a new production organization mode, virtual manufacturing cell is different from the general dynamic cell organization with reconfigurable physical location of machines, which only needs to carry out logical reconfiguration of machines in the planning and controlling system, without physical location reconfiguration ${ }^{[1-2]}$. It can be adapted to some enterprises, in which workshops with large, heavy, or immovable machines, that could meet the needs of multiple varieties, small-size batches, and multi-delivery periods.

There are many abnormal disturbances in the actual manufacturing process, such as emergency order insertion, machine failure, delivery date change or order quantity change, which lead to frequent adjustment of production plan, poor guidance of original plan and even lead to the disconnection between production plan and reality. Among them, the machine failure is one of the most common disturbance factors in the production system. The problem of production stagnation caused by the machine failure will lead to the failure of the original production process on the machines, so that adjusting the original scheduling scheme is necessary.

At present, many scholars have proposed many effective disturbance repairing methods to solve the occurrence of machine failure in the production system. In terms of model construction, Haleh ${ }^{[3]}$ considered factors such as lead time / delay penalty cost and failure maintenance time comprehensively in the construction of interruptible single machine rescheduling model under the condition of machine failure. $\mathrm{Ho}^{[4]}$ combined with the characteristics of textile and printed circuit board industries, constructed an unrelated parallel machine dynamic scheduling model considering the occurrence of machine failure. Han ${ }^{[5]}$ established a multi-objective rescheduling model of large-scale flow shop considering the blocking effect by taking machine failure as blocking condition. However, the above researches only consider the factors of production efficiency, but ignore the disturbance deviation due to the adjustment of schemes, which lead to a significant increase in scheduling costs ${ }^{[6]}$. In terms of algorithm design, Xiong ${ }^{[7]}$ designs a random adaptive search algorithm to solve the machine failure disturbance problem in the single machine environment. Wang ${ }^{[8]}$ proposes a hybrid evaluation algorithm based on fuzzy logic to solve the order delivery problem of 
distributed replacement flow shop under the condition of machine failure. Dong ${ }^{[9]}$ designs two new heuristic algorithms to solve job shop dynamic scheduling problem under failure disturbance. Weighted combination and Pareto solution are common methods to solve multi-objective programming problems. However, due to the coupling and conflict relationships among the different objectives, which is difficult to accurately reflect the objective relationships among them by assigning weights subjectively. Therefore, it is necessary to consider multiple-objectives in parallel in optimization $^{[5]}$.

In order to maintain the efficiency and stability of the production system, some scholars have adopted the robust-reactive ${ }^{[10]}$ method to formulate the rescheduling scheme by comprehensive consideration of the impacts of rescheduling scheme on the production activities in workshop. Akkan ${ }^{[11]}$ constructed a dual-objective single machine robust rescheduling model to minimize makespan and deviation between processing start time for the urgent order insertion problem. Valledor ${ }^{[12]}$ proposed a robust rescheduling method for flow shop, which aims to minimize the makespan, total weighted tardiness and operation deviation. The existing researches have following shortages: Firstly, the existing researches are generally carried out under the assumption that rescheduling is determined to occur, and the discussions of rescheduling trigger-decision mechanism have been ignored. Secondly, the existing researches pay more attention to the improvement of models, algorithms and its application in specific scenarios, but the applicable conditions and scope of the proposed methods are not clearly defined.

In conclusion, this paper combines transfer rescheduling and complete rescheduling strategies in the case of random disturbances of virtual manufacturing cell machine failure, constructs the cost evaluation function of rescheduling scheme based on the systematic analysis of rescheduling cost, and makes decisions on rescheduling time and strategy selection, so as to determine the optimal rescheduling strategy in the current situation In the deployment phase, a virtual manufacturing cell rescheduling model with the goal of minimizing makespan and equipment adjustment disturbance deviation is constructed, and which is solved by NSGA-II algorithm. Finally, an example is given to verify the feasibility and effectiveness of the proposed method, so as to realize the optimal control of the production system after the occurrence of machine failure.

\section{PROBLEM DESCRIPTION MATHEMATICAL MODELLING}

AND

\subsection{Problem Description}

The rescheduling problem of virtual manufacturing cell could be described as follows: At the beginning of production, there are $n$ workpieces to be processed in the workshop, the corresponding batch number of various workpieces is $B_{i}$; each workpiece contains $N_{i}$ operations; there are in total $m$ machines available to be selected in the cell. Suppose that the machine $k$ fails at $T=M B T$, and the estimated maintenance time is $M B R T$.

For better research on the rescheduling problem of virtual manufacturing cell, some assumptions are made as follows: (1) In the process of rescheduling, not all machines and workpieces are available immediately. Based on the premise that the processing task can not be interrupted, which means only when the machine completes the processing task of the current operation that it can continue to process the next operation; (2) According to the demands of the production process, the strict sequence between different operations only exist in the same kind of workpieces, and operations belonging to the different workpieces have the same priority; (3) Each operation could be processed on different machines, but one machine can only process one operation at the same time; (4) The same operation can only be carried out on one machine at the same time; (5) Without considering the situation of batch partition.

The related parameter symbols and definitions used in the model construction are as follows:

\section{(1) Input parameters}

$i-$ Index for parts, $i=1,2,3, \ldots, n$

$j-$ Index for operations, $j=1,2,3, \ldots, N_{i}$

$k$-Index for machines, $k=1,2,3, \ldots, m$

$O_{i, j}-$ Index for operations belonging to part $i$

(2) Parameter setting

$P T_{i, j} \longrightarrow$ Processing time of the $\mathrm{j}$ th operation of part $i$

$S T_{i, j} \longrightarrow$ Processing start time of the $\mathrm{jth}$ operation of part $i$

$E T_{i, j} \longrightarrow$ Processing end time of the $\mathrm{j}$ th operation of part $i$

$B_{i} \longrightarrow$ The batch size for part $i$

$L-$ An infinity positive number

$O_{i, j}^{M B}$ The set of operations to be scheduled affected by disturbance events

$M_{i, j}-$ The set of available machines 
corresponding to the $\mathrm{jth}$ operation of part $i$

(3) Decision variables

$P_{i, j, k}$ - If machine $M_{k}$ is available for processing operation $O_{i, j}, P_{i, j, k}=1$; otherwise, $P_{i, j, k}=0$

$Q_{i, j, k} \longrightarrow$ If machines that correspond to operation $O_{i, j}$ change, $Q_{i, j, k}=1$; otherwise, $Q_{i, j, k}=0$

$R_{i^{\prime}, j^{\prime}, k-i, j, k}$ - If operation $O_{i^{\prime}, j^{\prime}}$ is processed on machine $M_{k}$ immediately behind operation $O_{i, j}$, $R_{i^{\prime}, j^{\prime}, k-i, j, k}=1$; otherwise, $R_{i^{\prime}, j^{\prime}, k-i, j, k}=0$.

\subsection{Construction of Virtual Manufacturing Cell Rescheduling Model}

In this paper, on the basis of considering the rescheduling efficiency and disturbance deviation, a virtual manufacturing cell rescheduling model is proposed that based on the machine and part level.

\subsubsection{Objective Function}

(1) Minimum disturbance deviation of machine adjustment

$$
Q_{i, j, k}=\left\{\begin{array}{cc}
1 & P_{i, j, k}=P_{i, j, k}^{\prime} \\
0 & P_{i, j, k} \neq P_{i, j, k}^{\prime}
\end{array} \quad \forall i, j\right.
$$

(1)

According to Equation (1), the change of machines in the process procedure is judged, and the disturbance deviation caused by machine adjustment is shown in Equation (2).

$$
\text { PCDm }=\sum_{i=1}^{n} \sum_{j=1}^{N_{i}} \sum_{k=1}^{m} Q_{i, j, k}
$$

Therefore, the objective function construction based on the disturbance deviation of equipment adjustment is expressed as follows.

$$
f_{1}=\min \{P C D m\}_{\forall i, j, k}
$$

(2) Minimum makespan

$$
f_{2}=\min \left\{\max _{1 \leq i \leq n} C_{\max }\right\}
$$

In Equation (4), represents the makespan of parts in the manufacturing system, which means the complete time of the last operation of parts.

\subsubsection{Restrictions}

In Equation (4), represents the makespan of parts in the manufacturing system, which means the complete time of the last operation of parts.

$$
\begin{aligned}
& C_{\max } \geq S_{i, 1}+\sum_{j=1}^{N_{i}} P T_{i, j} \cdot B_{i} \quad \forall i \in(1,2, \ldots, n) \\
& S T_{i, j}+P T_{i, j} \cdot B_{i} \leq S T_{i,(j+1)} \\
& \forall i \in(1,2, \ldots, n), j \in\left(1,2, \ldots, N_{i}\right) \\
& E T_{i, j}=S T_{i, j}+P T_{i, j} \cdot B_{i} \\
& \forall i \in(1,2, \ldots, n), j \in\left(1,2, \ldots, N_{i}\right) \\
& S T_{i, j}+\left(1-R_{i^{\prime}, j^{\prime}, k-i, j, k}\right) \cdot L \geq S T_{i^{\prime} j^{\prime}}+P T_{i^{\prime} j^{\prime}} \\
& \forall i, i^{\prime} \in(1,2, \ldots, n), j, j^{\prime} \in\left(1,2, \ldots, N_{i}\right), \\
& k \in(1,2, \ldots, m) \\
& \sum_{k=1}^{m} P_{i, j, k}=1 \\
& \forall i \in(1,2, \ldots, n), j \in\left(1,2, \ldots, N_{i}\right) \\
& \sum_{k=1}^{m} R_{i^{\prime}, j^{\prime}, k-i, j, k}=1 \\
& \forall i \in(1,2, \ldots, n), j \in\left(1,2, \ldots, N_{i}\right) \\
& S T_{i, j} \geq 0, E T_{i, j} \geq 0, P T_{i, j} \geq 0 \\
& \forall i \in(1,2, \ldots, n), j \in\left(1,2, \ldots, N_{i}\right)
\end{aligned}
$$

Equation (5) represents the constraint on the maximum completion time of workpiece processing, that is, the makespan should be larger than or equal to the processing time of all parts. Equation (6) indicates that the sequence between operations of part must meet the constraints of the processing route. Equation (7) represents the corresponding relationships among the processing time, start time and completion time of each part. Equation (8) ensures that each machine can only process one process at the same time. Equation (9) indicates that any process can only be processed on one available device. Equation (10) indicates that there is and only one process immediately preceding any process. Equation (11) represents the non-negative constraints on the parameter variables in the model.

\subsection{NSGA-II Algorithm Design}

NSGA-II (Fast Elitist Non-Dominated Sorting Genetic Algorithm with Elite Strategy) algorithm effectively reduces the computational complexity of the algorithm by introducing strategies, such as fast nondominated sorting, elite selection, and crowding distance calculation, which has good performance in solving multi-objective optimization problems and been widely used in the research of scheduling problems. Therefore, this paper combines the characteristics of the problem 
and uses the NSGA-II algorithm to solve the problem.

\subsubsection{Encoding}

In order to describe the sequence of parts and machines simultaneously, in this paper, a double-layer real number structure based on the workpiece serial number and the equipment serial number is used to encode the chromosome. The first layer is part code, the number of occurrences of the serial number of parts represents the corresponding operations. The second layer is machine code, the number of occurrences of the serial number of machines represents the corresponding available machines.

\subsubsection{Selection, Crossover and Mutation Operations}

\section{(1) Selection operation}

In order to improve the efficiency of the solution, this paper sorts the population based on the non-dominated level of different individuals and calculates the crowding distance between different individuals, and on this basis, using the binary tournament selection method to select the population.

\section{(2) Cross operation}

Precedence operation crossover (POX) method is used to cross the part code and machine code in the chromosome. First, randomly select the position of the workpiece on the two parent chromosomes $\mathrm{P}_{1}$ and $\mathrm{P}_{2}$, and directly inherit the corresponding position gene to the children chromosomes $\mathrm{C}_{1}$ and $\mathrm{C}_{2}$. Then delete the inherited gene from the parent chromosome $\mathrm{P}_{1}$. Finally, distribute the remaining genes from parent chromosome $\mathrm{P}_{2}$ to the remaining positions of the child chromosome $\mathrm{C}_{1}$ in turn.

\section{(3) Mutation operation}

The single-point method and double-point method are used to perform mutation operations for machine code and part code respectively. The single-point method means selecting a gene on the corresponding chromosome of machine code as the mutation point, and randomly selecting another machine to replace the machine at the mutation point. The double-point method means selecting two genes on the chromosome randomly as the mutation points, and then exchanging the corresponding part code to generate new part code.

\section{RESCHEDULING STRATEGY FORMULATION FOR VIRTUAL MANUFACTURING CELL}

\subsection{Selection of Evaluation Index for Virtual Manufacturing Cell Rescheduling Scheme}

(1) Punctuality

In this paper, Equation (12) is used to evaluate the penalty cost of part $i$ under different non-on-time delivery situations.

$$
P C W_{i}=\left\{\begin{array}{lr}
\theta \cdot\left(t_{o t}-t_{i}\right) \cdot B_{i} & t_{i} \leq t_{o t} \\
\eta \cdot\left(t_{i}-t_{o t}\right) \cdot B_{i} & t_{o t}<t_{i} \leq t_{d t} \\
{\left[\eta \cdot\left(t_{d t}-t_{o t}\right)+\mu \cdot\left(t_{i}-t_{d t}\right)\right] \cdot B_{i}} & t_{d t}<t_{i}
\end{array}\right.
$$

The total penalty cost could be calculated as follows.

$$
P C W=\sum_{i=1}^{n} P C W_{i}
$$

In Equation (12), $t_{\text {ot }}$ represents the estimated completion time of part $i$ determined according to the initial scheduling plan, $t_{i}$ represents the actual completion time of part $i$ determined according to the re-scheduling plan, and $t_{t d}$ represents the latest time of part $i$ determined according to the production order information delivery time. $\theta, \eta, \mu$ respectively correspond to the early/tardiness penalty coefficients in the case of not exceeding the estimated completion time, not exceeding the latest delivery time, and exceeding the latest delivery time.

\section{(2) Stability}

The total penalty cost caused by the deviation of the processing start time before and after the scheme adjustment is shown in equation (14).

$$
P D E=\sum_{i=1}^{n} \sum_{j=1}^{N_{i}}\left|N S T_{i, j}-O S T_{i, j}\right| \cdot \sigma_{t}
$$

Where, $N S T_{i, j}$ represents the processing start time of the $j$ th operation of part $i$ in the rescheduling scheme; $O S T_{i, j}$ represents the processing start time of the $\mathrm{jth}$ operation of part $i$ in the original scheme. $\sigma_{t}$ represents the penalty coefficient for changes in the processing start time between the new and old schemes.

The final cost evaluation function of the rescheduling scheme is shown in Equation (15).

$$
P C=\delta \cdot P C W+(1-\delta) \cdot P D E
$$

In order to avoid the occurrence of rescheduling so frequently that the scheme cannot effectively guide the 
actual production, this paper will make decisions on the time of rescheduling and the choice of strategy based on equation (16).

$$
R S C=\min \left\{\begin{array}{l}
\min \left\{P C_{\text {part }}, P C_{\text {initial }}\right\} \\
\min \left\{P C_{\text {comp }}, P C_{\text {initial }}\right\}
\end{array}\right\}
$$

\subsection{Rescheduling Decision Method for Virtual Manufacturing Cell}

According to the above contents, the specific implementation steps of the proposed virtual cell rescheduling method are as follows:

Step1: Achieve the real-time information of parts, machines and orders in the cell.

Step2: Based on the real-time information in the cell, the initial scheduling scheme is obtained by genetic algorithm with the goal of minimizing the maximum completion time.

Step3: Executing the scheme and monitors the execution of the scheduling scheme. If there is a disturbance event, go to Step4; otherwise, continue to execute the current scheme.

Step4: When the equipment failure occurs, input the information of the failure machine in the scheduling system, determine the serial number, layout and location of the failure machine, and estimate the failure maintenance time.

Step5: According to the current failure information, the corresponding rescheduling scheme is generated based on the transfer rescheduling strategy and the complete rescheduling strategy, and the rescheduling occurrence time and strategy selection are decided according to the cost evaluation function of the rescheduling scheme.

Step6: According to the selection result of the rescheduling scheme, input the scheduling information, update and execute the rescheduling scheme, and go to Step3.

\section{NUMERICAL EXPERIMENT}

\subsection{Basic Data}

In this paper, based on the numerical data, the proposed virtual manufacturing cell rescheduling method is applied to optimization. In a certain period, there are multiple orders arriving. According to the order demand, the workshop needs to process 8 kinds of parts, and each kind of parts contains multiple processes. There are four processing areas in the workshop, and eight optional machines are evenly distributed in the workshop. Refer Table 1 and Table 2 to more information of machines, operations, processing time and delivery time.

Table 1 Corresponding processing time of each operation in cells

\begin{tabular}{|c|c|c|c|c|c|}
\hline \multicolumn{2}{|c|}{ Production Information } & \multicolumn{5}{|c|}{ Processing Time(h) } \\
\hline Number & Batch & Operation 1 & Operation 2 & Operation 3 & Operation 4 \\
\hline$P_{1}$ & 6 & 7.1 & 8.3 & 6.7 & 7.4 \\
\hline$P_{2}$ & 4 & 6.4 & 1.4 & 3.5 & 4.8 \\
\hline$P_{3}$ & 3 & 12.6 & 11.4 & 7.4 & 9.5 \\
\hline$P_{4}$ & 5 & 15 & 11 & 8.7 & $/$ \\
\hline$P_{5}$ & 2 & 7.8 & 4.6 & 9.1 & $/$ \\
\hline$P_{6}$ & 6 & 6.6 & 7.8 & 9.6 & $/$ \\
\hline$P_{7}$ & 7 & 6.9 & 7.5 & 8.4 & $/$ \\
\hline$P_{8}$ & 3 & 5.2 & 3.3 & 5.4 & \\
\hline
\end{tabular}

Table 2 Available machines for each operation and delivery time

\begin{tabular}{|c|c|c|c|c|c|c|}
\hline \multicolumn{2}{|c|}{ Production Information } & \multicolumn{4}{|c|}{ Available Machines } & \multirow{2}{*}{ Delivery Time } \\
\cline { 1 - 5 } Number & Batch & Operation 1 & Operation 2 & Operation 3 & Operation 4 & \multirow{2}{*}{200} \\
\hline$P_{1}$ & 6 & {$[1,5,6]$} & {$[2,3]$} & {$[4,7]$} & {$[8]$} & 180 \\
\hline$P_{2}$ & 4 & {$[2,6]$} & {$[1,3]$} & {$[5,7]$} & {$[4,8]$} & 180 \\
\hline$P_{3}$ & 3 & {$[1,2]$} & {$[6,7]$} & {$[3,4,8]$} & {$[5]$} & 180 \\
\hline$P_{4}$ & 5 & {$[5,6]$} & {$[2,8]$} & {$[1,4,7]$} & $/$ & 180 \\
\hline$P_{5}$ & 2 & {$[1,2,3]$} & {$[5,6]$} & {$[4,7,8]$} & $/$ & 180 \\
\hline$P_{6}$ & 6 & {$[2,4]$} & {$[1,6]$} & {$[5,8]$} & {$[3,7]$} & \\
\hline
\end{tabular}




\begin{tabular}{|l|l|c|c|c|c|c|}
\hline$P_{7}$ & 7 & {$[1,3,4]$} & {$[2,5]$} & {$[6,7,8]$} & $/$ & 180 \\
\hline$P_{8}$ & 3 & {$[3,7]$} & {$[1,4]$} & {$[2,5]$} & $/$ & 165 \\
\hline
\end{tabular}

\subsection{Data Analysis}

\subsubsection{Generating Initial Scheduling Scheme of Virtual Manufacturing Cell}

According to the relevant information in Section 4.1, this paper takes minimizing makespan as optimization objective and uses genetic algorithm to schedule processing tasks in order, and then generates the initial scheduling scheme of virtual manufacturing cell, the results just shown in Table 3 .

\subsubsection{Generating rescheduling scheme of virtual manufacturing cell}

The NSGA-II algorithm proposed in this paper is implemented by MATLAB 2016a, and the algorithm parameters are set as follows: The total number of chromosomes is 80 , the probability of crossover between workpiece code and machine code is 0.8 , the probability of mutation is 0.2 , the upper limit of algorithm iterations is 200 , the initial population is generated randomly, and the termination condition is to achieve the predetermined number of iterations.

The operation parameters are set as follows: The value of the corresponding penalty coefficients $\theta, \eta$, $\mu$ are 50,50, 100 respectively. The penalty coefficient $\sigma_{t}$ is 100 , and the weight coefficient $\delta$ is 0.5 .

At the moment of $T=120$, the machine $M_{4}$ fails down and the estimated maintenance time is 25 hours. Three strategies are implemented to deal with consequences caused by machine failure through adjusting operations.

Table 3 Initial scheduling scheme of virtual cell

\begin{tabular}{|c|c|c|c|c|c|}
\hline \multicolumn{4}{|c|}{ Production Information } & \multicolumn{4}{|c|}{ Machines ( Processing start time-Processing end time, Unit: $h$ ) } \\
\hline Number & Batch & Operation 1 & Operation 2 & Operation 3 & Operation 4 \\
\hline$P_{1}$ & 6 & $M_{6}(0-42.6)$ & $M_{3}(48.3-98.1)$ & $M_{4}(98.1-138.3)$ & $M_{8}(138.3-182.7)$ \\
\hline$P_{2}$ & 4 & $M_{2}(37.8-63.4)$ & $M_{3}(113.7-119.3)$ & $M_{7}(119.3-133.3)$ & $M_{4}(138.3-157.5)$ \\
\hline$P_{3}$ & 3 & $M_{2}(0-37.8)$ & $M_{7}(37.8-72)$ & $M_{4}(72-94.2)$ & $M_{5}(144-172.5)$ \\
\hline$P_{4}$ & 5 & $M_{5}(0-75)$ & $M_{8}(75-130)$ & $M_{1}(130-173.5)$ & $/$ \\
\hline$P_{5}$ & 2 & $M_{1}(86.4-102)$ & $M_{6}(102-111.2)$ & $M_{4}(157.5-175.7)$ & $/$ \\
\hline$P_{6}$ & 6 & $M_{4}(0-39.6)$ & $M_{1}(39.6-86.4)$ & $M_{5}(86.4-144)$ & $M_{3}(144-188.4)$ \\
\hline$P_{7}$ & 7 & $M_{3}(0-48.3)$ & $M_{2}(63.4-115.9)$ & $M_{6}(115.9-174.7)$ & $/$ \\
\hline$P_{8}$ & 3 & $M_{3}(98.1-113.7)$ & $M_{1}(113.7-123.6)$ & $M_{2}(123.6-139.8)$ & $/$ \\
\hline
\end{tabular}

The adjustment of the original production plan will lead to the advance / delay of the delivery time of some products and the change of the processing start time of some processes. In view of the above disturbance deviation, this paper makes decision on the occurrence time and strategy selection of rescheduling according to the cost evaluation function of rescheduling scheme, and the indicators obtained are shown in Table 4.

Table 4 Performance comparison of different schemes

\begin{tabular}{|c|c|c|c|}
\hline Evaluating Indicator & $\begin{array}{c}\text { Maintaining } \\
\text { Scheme }\end{array}$ & $\begin{array}{c}\text { Transfer Rescheduling } \\
\text { Scheme }\end{array}$ & $\begin{array}{c}\text { Complete Rescheduling } \\
\text { Scheme }\end{array}$ \\
\hline Disturbance repair cost & 6330 & 3130 & 2020 \\
\hline
\end{tabular}




\begin{tabular}{|l|l|l|l|}
\hline Makespan & 207.7 & 207.7 & 196 \\
\hline
\end{tabular}

According to Table 4, rescheduling needs to be performed when the machine failure occurs. Through the comparative analysis of the performance of various rescheduling schemes, it is found that in terms of rescheduling cost, the costs of transfer rescheduling scheme and complete rescheduling scheme are both lower than maintaining scheme. Furthermore, the cost of complete rescheduling scheme is $35.5 \%$ lower than that of transfer rescheduling scheme. In terms of makespan, the complete rescheduling scheme is better than the transfer rescheduling scheme that shortens 11.7 hours, so the complete rescheduling scheme is more suitable for this kind of disturbance.

\subsection{Analysis of different failure disturbance}

In order to verify the effectiveness of the proposed rescheduling method under different disturbances, this paper applies the proposed method to nine types of random disturbances which occur at different times and have different maintenance durations, and the specific scheduling optimization and selection decision-making process is the same as the previous one The results are shown in Table 5.

According to Table 6, it can be found that the transfer rescheduling scheme can show better performance in the case of short maintenance time or late failure occurrence time, while the complete rescheduling scheme is more suitable in the case of long maintenance time or early failure occurrence time. The above results show that the combination of multiple rescheduling strategies can make up for the defects of using a single method, and ensure the rationality and economy of the rescheduling scheme while maintaining the continuity of cell production.

\section{CONCLUSION}

In this paper, the virtual cell rescheduling problem under random disturbance of equipment failure is studied, and a virtual cell rescheduling method based on multiple strategy combinations is proposed. Based on the systematic analysis of rescheduling cost factors, this paper constructs the cost evaluation function of rescheduling scheme, and constructs the virtual cell rescheduling model based on this, and solves the model with NSGA-II algorithm. In order to determine the occurrence time and strategy selection of rescheduling according to the cost evaluation function whenever disturbance occurs, the rationality and economy of the proposed rescheduling method are verified by a numerical example. The results show that the combination of multiple rescheduling strategies can maintain the applicability of the rescheduling scheme under different disturbances, improve the scheduling efficiency, reduce the disturbance deviation and reduce the scheduling cost.

The existing research on rescheduling method is based on the premise that the processing cannot be interrupted. In the follow-up research work, we can further study the situation that the workpiece processing is allowed to be interrupted under the condition of equipment failure, so that the rescheduling scheme is more in line with the actual production. At the same time, the rescheduling strategies suitable for different equipment fault disturbance situations can be deeply mined, and the method can be extended to other disturbance situations.

Table 5 Performance comparison of different schemes under various failure disturbance

\begin{tabular}{|c|c|c|c|c|c|c|c|c|}
\hline \multirow{2}{*}{ Number } & \multirow{2}{*}{$\begin{array}{l}\text { Moment of } \\
\text { failures } \\
\text { occur (h) }\end{array}$} & \multirow{2}{*}{$\begin{array}{l}\text { Maintenance } \\
\text { time (h) }\end{array}$} & \multicolumn{2}{|c|}{ Maintaining Scheme } & \multicolumn{2}{|c|}{$\begin{array}{l}\text { Transfer Rescheduling } \\
\text { Scheme }\end{array}$} & \multicolumn{2}{|c|}{$\begin{array}{l}\text { Complete Rescheduling } \\
\text { Scheme }\end{array}$} \\
\hline & & & $\begin{array}{l}\text { Disturbance } \\
\text { repair cost }\end{array}$ & $\begin{array}{l}\text { Makespan } \\
\text { (h) }\end{array}$ & $\begin{array}{l}\text { Disturbance } \\
\text { repair cost }\end{array}$ & $\begin{array}{c}\text { Makespan } \\
\text { (h) }\end{array}$ & $\begin{array}{l}\text { Disturbance } \\
\text { repair cost }\end{array}$ & $\begin{array}{c}\text { Makespan } \\
\text { (h) }\end{array}$ \\
\hline 1 & \multirow{3}{*}{$\mathrm{U}(40,60)$} & $U(5,15)$ & 1735 & 194.7 & 560 & 188.4 & 645 & 192.6 \\
\hline 2 & & $U(15,25)$ & 2365 & 192.3 & 1405 & 191.4 & 1585 & 199.2 \\
\hline 3 & & $U(25-35)$ & 4255 & 205.2 & 2680 & 202.2 & 2575 & 204.8 \\
\hline 4 & \multirow{3}{*}{$U(80,100)$} & $U(5,15)$ & 1145 & 193.6 & 575 & 188.4 & 1030 & 194.3 \\
\hline 5 & & $U(15,25)$ & 2480 & 199.5 & 2605 & 198.5 & 2525 & 199.1 \\
\hline 6 & & $U(25-35)$ & 5655 & 208.4 & 3160 & 203.4 & 2530 & 202.6 \\
\hline 7 & $U(120,140)$ & $U(5,15)$ & 875 & 193.5 & 875 & 188.4 & 1235 & 193.5 \\
\hline
\end{tabular}




\begin{tabular}{|l|l|l|l|l|l|l|l|l|}
\hline 8 & \multirow{2}{*}{9} & $\mathrm{U}(15,25)$ & 3795 & 198.7 & 2885 & 200.6 & 2035 & 199.1 \\
\cline { 3 - 9 } & & $\mathrm{U}(25-35)$ & 6330 & 207.8 & 3130 & 207.7 & 2020 & 196 \\
\hline
\end{tabular}

Table 6 Rescheduling scheme selection under random machine failure disturbance

\begin{tabular}{|c|c|c|c|c|}
\hline Number & $\begin{array}{c}\text { Moment of failures } \\
\text { occur (h) }\end{array}$ & Maintenance time (h) & $\begin{array}{l}\text { Trigger } \\
\text { rescheduling }\end{array}$ & Rescheduling scheme selection \\
\hline 1 & \multirow{3}{*}{$U(40,60)$} & $U(5,15)$ & $\sqrt{ }$ & Transfer Rescheduling \\
\hline 2 & & $U(15,25)$ & $\sqrt{ }$ & Transfer Rescheduling \\
\hline 3 & & $U(25-35)$ & $\sqrt{ }$ & Complete Rescheduling \\
\hline 4 & \multirow{3}{*}{$\mathrm{U}(80,100)$} & $U(5,15)$ & $\sqrt{ }$ & Transfer Rescheduling Scheme \\
\hline 5 & & $U(15,25)$ & $x$ & / \\
\hline 6 & & $U(25-35)$ & $\sqrt{ }$ & Complete Rescheduling \\
\hline 7 & \multirow{3}{*}{$U(120,140)$} & $U(5,15)$ & $x$ & / \\
\hline 8 & & $U(15,25)$ & $x$ & / \\
\hline 9 & & $U(25-35)$ & $\sqrt{ }$ & Complete Rescheduling \\
\hline
\end{tabular}

\section{REFERENCES}

[1] Jayachitra, R., \& Prasad, P. S. S. (2011). Performance analysis of virtual cellular manufacturing: a simulation study. International Journal of Services and Operations Management, 8(1), 92-107.

[2] McLean, C. R., Bloom, H. M., \& Hopp, T. H. (1983). The virtual manufacturing cell. In Information Control Problems in Manufacturing Technology 1982 (pp. 207-215). Pergamon.

[3] Haleh, H., Maghsoudlou, H., Hadipour, H., \& Nabovati, H. (2017). Scheduling single machine with random breakdown and preemptive jobs. Journal of Industrial and Production Engineering, 34(4), 289-299.

[4] Kim, Y. H., \& Kim, R. S. (2020). Insertion of new idle time for unrelated parallel machine scheduling with job splitting and machine breakdowns. Computers \& Industrial Engineering, 147, 106630.

[5] Han, Y., Gong, D., Jin, Y., \& Pan, Q. (2017). Evolutionary multiobjective blocking lot-streaming flow shop scheduling with machine breakdowns. IEEE transactions on cybernetics, 49(1), 184-197.

[6] Tolio, T., Urgo, M., \& Váncza, J. (2011). Robust production control against propagation of disruptions. CIRP annals, 60(1), 489-492.

[7] Xiong, X., Wang, D., Edwin Cheng, T. C., Wu, C. C., \& Yin, Y. (2018). Single-machine scheduling and common due date assignment with potential machine disruption. International Journal of Production Research, 56(3), 1345-1360.
[8] Wang, K., Huang, Y., \& Qin, H. (2016). A fuzzy logic-based hybrid estimation of distribution algorithm for distributed permutation flowshop scheduling problems under machine breakdown. Journal of the Operational Research Society, 67(1), 68-82.

[9] Dong, Y. H., \& Jang, J. (2012). Production rescheduling for machine breakdown at a job shop. International Journal of Production Research, 50(10), 2681-2691.

[10] Minguillon, F. E. \& Stricker, N. (2020). Robust predictive-reactive scheduling and its effect on machine disturbance mitigation. CIRP Annals, 69(1), 401-404.

[11] Akkan, C. (2015). Improving schedule stability in single-machine rescheduling for new operation insertion. Computers \& Operations Research, 64, 198-209.

[12] Valledor, P., Gomez, A., Priore, P., \& Puente, J. (2018). Solving multi-objective rescheduling problems in dynamic permutation flow shop environments with disruptions. International Journal of Production Research, 56(19), 6363-6377. 